\title{
ON $r$-SEPARATED SETS IN NORMED SPACES
}

\author{
JUAN ARIAS-DE-REYNA
}

(Communicated by Andrew M. Bruckner)

\begin{abstract}
The separation of a bounded set $A$ in a metric space $\delta(A)$ is defined as the supremum of the numbers $r>0$ such that there exists a sequence $\left(x_{n}\right)$ in $A$ such that $d\left(x_{n}, x_{m}\right)>r$ for every $n \neq m$. We prove for every bounded set $A$ in a Banach space that $\delta(A)=\delta(\operatorname{co}(A))$ where $\operatorname{co}(A)$ denotes the convex hull of $A$. This yields a generalization of Darbo's fixed point theorem.
\end{abstract}

\section{INTRODUCTION}

In 1939 Kuratowski [10] introduced the measure of noncompactness $\alpha(A)$ of a bounded set $A$ in a metric space $X, \alpha(A)$ is called the Kuratowski measure of noncompactness, and is defined as the greatest lower bound of the numbers $r>0$ such that $A$ can be decomposed into a finite union of sets of diameter smaller than $r$. The condition $\alpha(A)=0$ therefore means that $A$ is precompact. Another measure of noncompactness, which in many cases seems to be more convenient, is called the ball-measure, $\beta(A)$, and is defined as the infimum of the real numbers $r>0$ such that there is a finite cover of $A$ with balls of radii smaller than $r$.

These and other measures of noncompactness were used by Darbo [2], Massat [11], Sadovskii [12], and Banaś and Goebel [1] to obtain some fixed point theorems of nonlinear maps. In general, a measure of noncompactness on a complete metric space $X$ is a function $\gamma$ which maps every bounded set $B \subset X$ to a positive real number $\gamma(B)$ such that:

(a) $\gamma(B)=0$ if and only if $\bar{B}$ is compact;

(b) if $B \subset C$ are bounded sets, then $\gamma(B) \leq \gamma(C)$.

Furthermore, if $B$ is a closed convex bounded subset of a Banach space $X$, an operator $T: B \rightarrow B$ is called $\gamma$-condensing if for all bounded sets $C \subset \mathrm{B}$ we have $\gamma(T(C)) \leq \gamma(C)$ with equality if and only if $\gamma(C)=0$.

The following theorem (Sadovskii [12], Massat [11]) illustrates the utility of these concepts.

Received by the editors February 26, 1990.

1980 Mathematics Subject Classification (1985 Revision). Primary 46B20; Secondary 54E40. 
Theorem. Let $X$ be a Banach space and $B \subset X$ a closed convex bounded set. Let $T: B \rightarrow B$ be a continuous and $\gamma$-condensing operator, where $\gamma$ is a measure of noncompactness on $X$ such that

(c) $\gamma(A \cup B)=\gamma(A)$ for every finite set $B \subset X$;

(d) $\gamma(A)=\gamma(\operatorname{co}(A))$ for every bounded set $A \subset X$, where $\operatorname{co}(A)$ denotes the convex hull of $A$.

Then $T$ has a fixed point.

In Wells and Williams [13], another measure of noncompactness is defined: the separation of $A, \delta(A)$, is the supremum of the numbers $r>0$ such that there exists a sequence $\left(x_{n}\right)$ in $A$ such that $d\left(x_{n}, x_{m}\right)>r$ for every $n \neq$ $m$. They use it because this measure can distinguish between the unit balls of Banach spaces. The measure of the unit ball seems to be connected with the reflexivity of a Banach space (Kottman [9]). And the theorem of Elton and Odell [6] insures that $\delta(B)>1$ for the unit ball $B$ of every infinite dimensional Banach space.

This same concept of separation of $A$ has been defined independently by Dominguez Benavides [3] and is denoted by $\mu(A)$. He observes [4] that in some Banach spaces as the $l_{p}$ spaces $(1<p<+\infty), \delta(A)=\mu(A)$ is proportional to the $\beta$-measure. In other spaces, say $L^{p}[0,1](p \neq 2)$, this relation is not satisfied.

Observe that the above fixed point theorem would apply to this measure of noncompactness if we proved that

$$
\delta(A)=\delta(\operatorname{co}(A))
$$

for every bounded subset $A$ of a Banach space. The purpose of this paper is to prove this. It is important to point out that, in a recent paper, Dominguez Benavides [5] proves that every $\alpha$-contraction is a $\delta$-contraction, so the fixed point theorem obtained in our paper generalizes the fixed point theorem of Darbo [2].

In $\S 2$ we give a probabilistic lemma (Corollary 2) that plays an essential role in the proof of the main theorem (Theorem 5). In that proof we also use an easier version of a deep theorem of D. H. Fremlin and M. Talagrand about random graphs, which we enunciate as Theorem 3.

\section{Probabilistic lemmas}

Lemma 1. Let $\mu, \nu$ be two probability measures on the space $[-1,1]$ such that

$$
\int_{-1}^{1} x d \mu(x)-\int_{-1}^{1} x d \nu(x) \geq s>\theta>1
$$

then there exists a real number $t_{0} \in[\theta-1,1]$ such that

$$
\mu\left[-1, t_{0}\right)+\nu\left[t_{0}-\theta, 1\right] \leq(2-s) /(2-\theta) .
$$


Proof. Observe that an elementary calculation leads us to

$$
\int_{-1}^{1}(1+x) d \mu(x)=\int_{0}^{2} \mu[-1+t, 1] d t=\int_{-1}^{1} \mu[t, 1] d t .
$$

It follows that

$$
\begin{aligned}
& \int_{-1}^{1} x d \mu(x)=1-\int_{-1}^{1} \mu[-1, t) d t \\
& \int_{-1}^{1} x d \nu(x)=\int_{-1}^{1} \nu[t, 1] d t-1
\end{aligned}
$$

Therefore

$$
\begin{aligned}
\int_{-1}^{1} x d \mu(x)-\int_{-1}^{1} x d \nu(x) & =2-\int_{-1}^{1} \mu[-1, t)-\int_{-1}^{1} \nu[t, 1] d t \\
& =2-\int_{-1}^{1} \mu[-1, t)-\int_{-1+\theta}^{1+\theta} \nu[t-\theta, 1] d t \\
& \leq 2-\int_{-1+\theta}^{1}(\mu[-1, t)+\nu[t-\theta, 1]) d t
\end{aligned}
$$

Define $\psi(t)=(\mu[-1, t)+\nu[t-\theta, 1])$. By the hypothesis we know that

$$
2-s \geq \int_{-1+\theta}^{1} \psi(t) d t .
$$

Then $\psi(t)>(2-s) /(2-\theta)$ for every $t \in[-1+\theta, 1]$ would lead to a contradiction. It follows that there exists $t_{0} \in[-1+\theta, 1]$ such that $\psi\left(t_{0}\right) \leq$ $(2-s) /(2-\theta)$.

We shall use the following corollary in the proof of the main theorem.

Corollary 2. Let $\left(\Omega_{1}, \mathbb{P}_{1}\right),\left(\Omega_{2}, \mathbb{P}_{2}\right)$ be two probability spaces and $X_{i}: \Omega_{i} \rightarrow E$ two random variables with values in the same Banach space $E$. Assume that $\left\|X_{i}\right\|_{\infty} \leq 1$ and

$$
\left\|\mathbb{E}\left(X_{1}\right)-\mathbb{E}\left(X_{2}\right)\right\| \geq s>\theta>1 .
$$

Then there exist two measurable subsets $A \subset \Omega_{1}$ and $B \subset \Omega_{2}$ such that

$$
\begin{gathered}
\mathbb{P}_{1}(A)+\mathbb{P}_{2}(B) \leq(2-s) /(2-\theta)<1 . \\
\omega_{1} \notin A \text { and } \omega_{2} \notin B \text { implies }\left\|X_{1}\left(\omega_{1}\right)-X_{2}\left(\omega_{2}\right)\right\|>\theta .
\end{gathered}
$$

Proof. There is no loss of generality in assuming that the Banach space $E$ is real. Let $x^{*} \in E^{*}$ be a vector of the dual space such that $\left\|x^{*}\right\|=1$ and

$$
\left\|\mathbb{E}\left(X_{1}\right)-\mathbb{E}\left(X_{2}\right)\right\|=\mathbb{E}\left(x^{*} \circ X_{1}\right)-\mathbb{E}\left(x^{*} \circ X_{2}\right) .
$$

Let $\mu$ and $\nu$ be the image of the measures $\mathbb{P}_{1}$ and $\mathbb{P}_{2}$ under the mappings $x^{*} \circ X_{1}$ and $x^{*} \circ X_{2}$. Since $\left\|X_{i}\right\|_{\infty} \leq 1, \mu$ and $\nu$ are probability measures on $[-1,1]$. Observe that

$$
\int_{-1}^{1} x d \mu(x)-\int_{-1}^{1} x d \nu(x)=\mathbb{E}\left(x^{*} \circ X_{1}\right)-\mathbb{E}\left(x^{*} \circ X_{2}\right) \geq s>\theta>1 .
$$


Applying the lemma we find $t_{0} \in[\theta-1,1]$ such that

$$
\mu\left[-1, t_{0}\right)+\nu\left[t_{0}-\theta, 1\right] \leq(2-s) /(2-\theta)<1 .
$$

Define $A=\left(x^{*} \circ X_{1}\right)^{-1}\left[-1, t_{0}\right)$ and $B=\left(x^{*} \circ X_{2}\right)^{-1}\left[t_{0}-\theta, 1\right]$. Thus $A \subset \Omega_{1}$ and $B \subset \Omega_{2}$ are measurable sets that satisfy (1).

If $\omega_{1} \notin A$ and $\omega_{2} \notin B$, then $x^{*}\left(X_{1}\left(\omega_{1}\right)\right) \geq t_{0}$ and $x^{*}\left(X_{2}\left(\omega_{2}\right)\right)<t_{0}-\theta$. It follows that

$$
\left\|X_{1}\left(\omega_{1}\right)-X_{2}\left(\omega_{2}\right)\right\| \geq x^{*}\left(X_{1}\left(\omega_{1}\right)-X_{2}\left(\omega_{2}\right)\right)>\theta
$$

and (2) is also satisfied.

We shall use the following consequence of a theorem of D. H. Fremlin and M. Talagrand [7]:

Theorem 3. Let $\left(\Omega_{n}, \mathbb{P}_{n}\right)$ be a sequence of probability spaces. For every $m<n$, let $B_{n, m} \subset \Omega_{n}$ be a measurable set such that $\mathbb{P}_{n}\left(B_{n, m}\right) \leq \alpha<1$. Then there exists an infinite set $J \subset \mathbf{N}$ such that, for every $n \in J$,

$$
\mathbb{P}_{n}\left(\bigcup_{\substack{m<n \\ m \in J}} B_{n, m}\right)<1 .
$$

Proof. We apply the theorems $6 \mathrm{C}$ and $6 \mathrm{D}$ [7] to the probability space $\Omega=$ $\prod_{j=1}^{\infty} \Omega_{j}$ and the measurable sets

$$
E_{n, m}=\Omega \backslash\left(B_{n, m} \times \prod_{j \neq n} \Omega_{j}\right)
$$

in order to obtain the theorem.

\section{THEOREM ABOUT THE MEASURE OF NONCOMPACTNESS}

Let us recall that if $A \subset X$ is a bounded subset in a metric space $X$, the separation of $A, \delta(A)$ is defined as the supremum of the real numbers $r>0$ such that there exists a sequence $\left(x_{n}\right)$ in $A$ verifying $\left\|x_{n}-x_{m}\right\|>r$ for every two distinct elements $x_{n}, x_{m}$ of the sequence.

We begin making a reduction in the possible counterexample to $\delta(A)=$ $\delta(\operatorname{co}(A))$.

Proposition 4. Let $A$ be a bounded subset of the normed space $X$ such that $\delta(A)<\delta(\operatorname{co}(A))$. Then, for every s verifying $\delta(A)<s<\delta(\operatorname{co}(A))$, there exists a set $B$ contained in the ball of center 0 and radius $s$ and such that

$$
\delta(B) \leq \delta(A)<\delta(\operatorname{co}(A))=\delta(\operatorname{co}(B)) .
$$

Proof. Take a maximal set $\left\{a_{k}\right\}_{k=1}^{N}$ of points belonging to $A$ such that $i \neq j$ implies $\left\|a_{i}-a_{j}\right\| \geq s$. The number $N$ of elements is finite since $\delta(A)<s$. 
It is clear that $A$ is contained in the union of the balls $B\left(a_{k}, s\right)$ of center $a_{k}$ and radius $s$ :

$$
A \subset \bigcup_{k=1}^{N} B\left(a_{k}, s\right) .
$$

Every point $x \in \operatorname{co}(A)$ can be written in the form $x=\sum_{k=1}^{N} \alpha_{k} x_{k}$, where $\alpha_{k} \geq$ $0, \sum \alpha_{k}=1$ and $x_{k} \in \operatorname{co}\left(A \cap B\left(a_{k}, s\right)\right)$. Now, given $\varepsilon>0$, let $\left\{z_{n}\right\}_{n=1}^{\infty}$ be a sequence of points in $\operatorname{co}(A)$ such that $n \neq m$ implies $\left\|z_{n}-z_{m}\right\|>\delta(\operatorname{co}(A))-\varepsilon$.

Write every $z_{n}$ in the form:

$$
z_{n}=\sum_{k=1}^{N} \alpha_{k}^{n} y_{k}^{n}
$$

where $\alpha_{n}^{k} \geq 0, \sum_{k=1}^{N} \alpha_{k}^{n}=1$, and $y_{k}^{n} \in \operatorname{co}\left(A \cap B\left(a_{k}, s\right)\right)$. Choose an infinite subset $I \subset \mathbf{N}$ such that if $n, m \in I$, then for all $k \leq N$,

$$
\left|\alpha_{k}^{n}-\alpha_{k}^{m}\right|<\varepsilon /\left(N \cdot \sup _{a \in A}\|a\|\right) .
$$

Now let $n, m \in I$ and $n \neq m$. Then there exists a natural number $k$ such that $\left\|y_{k}^{n}-y_{k}^{m}\right\|>\delta(\operatorname{co}(A))-2 \varepsilon$, because otherwise

$$
\begin{aligned}
\left\|z_{n}-z_{m}\right\| & \leq\left\|\sum_{k=1}^{N} \alpha_{k}^{n}\left(y_{k}^{n}-y_{k}^{m}\right)+\left(\alpha_{k}^{n}-\alpha_{k}^{m}\right) y_{k}^{m}\right\| \\
& \leq(\delta(\operatorname{co}(A))-2 \varepsilon) \cdot 1+\sup _{a \in A}\|a\| \sum_{k=1}^{N}\left|\alpha_{k}^{n}-\alpha_{k}^{m}\right| \\
& <\delta(\operatorname{co}(A))-\varepsilon,
\end{aligned}
$$

which contradicts the hypothesis.

Applying the Ramsey theorem, T. Jech [8], we obtain an infinite set $J$ contained in $I$ and an index $k$ such that $n, m \in J, n \neq m$ imply $\left\|y_{k}^{n}-y_{k}^{m}\right\| \geq$ $\delta(\operatorname{co}(A))-2 \varepsilon$. Therefore, for every $\varepsilon>0$, there exists an index $k, 1 \leq k \leq N$, such that $\delta\left(\operatorname{co}\left(A \cap B\left(a_{k}, s\right)\right)\right) \geq \delta(\operatorname{co}(A))-2 \varepsilon$. Hence there exists an index $k$ such that

$$
\delta\left(\operatorname{co}\left(A \cap B\left(a_{k}, s\right)\right)\right) \geq \delta(\operatorname{co}(A)) .
$$

Furthermore we observe that $\delta\left(A \cap B\left(a_{k}, s\right)\right) \leq \delta(A)$. We can translate the set $A \cap B\left(a_{k}, s\right)$ and obtain the set $B=-a_{k}+A \cap B\left(a_{k}, s\right)$, which is contained in the ball of center 0 and radius $s$ and satisfies

$$
\delta(B) \leq \delta(A)<\delta(\operatorname{co}(A)) \leq \delta(\operatorname{co}(B)) .
$$

Theorem 5. Let $A \subset X$ be a bounded subset of a normed space $X$. Then $\delta(A)=\delta(\operatorname{co}(A))$.

Proof. Suppose that the theorem is false. Then Proposition 4 implies that there exists a subset $A$ of the unit ball of a Banach space $E$, such that

$$
\delta(A)<1<s<\delta(\operatorname{co}(A)) .
$$


We can take a sequence $\left(x_{n}\right)$ in $\operatorname{co}(A)$ such that for every $n \neq m,\left\|x_{n}-x_{m}\right\| \geq$ $s$. As $x_{n} \in \operatorname{co}(A)$, there exists a finite subset $\Omega_{n} \subset A$ and, for every $e \in \Omega_{n}$, a real number $\alpha_{e}>0$ such that

$$
x_{n}=\sum_{e \in \Omega_{n}} \alpha_{e} e \text { and } \sum_{e \in \Omega_{n}} \alpha_{e}=1 .
$$

Let $\mathbb{P}_{n}$ be the probability defined on $\Omega_{n}$ by

$$
\mathbb{P}_{n}(B)=\sum_{e \in B} \alpha_{e}
$$

for every $B \subset \Omega_{n}$.

For every $n$, let $X_{n}: \Omega_{n} \rightarrow E$ be the random variable, defined as the identity in $\Omega_{n}$. It is clear that if we choose $\theta$ such that $1<\theta<s$, we have, for every $n \neq m$,

$$
\left\|\mathbb{E}\left(X_{n}\right)-\mathbb{E}\left(X_{m}\right)\right\|=\left\|x_{n}-x_{m}\right\|>s>\theta>1 .
$$

We are now in a position to apply our probabilistic lemma and find subsets $B_{n, m} \subset \Omega_{n}$ and $B_{m, n} \subset \Omega_{m}$ verifying:

(a) $\mathbb{P}_{n}\left(B_{n, m}\right)+\mathbb{P}_{m}\left(B_{m, n}\right) \leq(2-s) /(2-\theta)<1$.

(b) $e_{n} \notin B_{n, m}$ and $e_{m} \notin B_{m, n}$ implies $\left\|e_{n}-e_{m}\right\| \geq \theta$.

For every natural number $m_{0},\left(B_{m_{0}, n}\right)_{n>m_{0}}$ is a sequence of subsets of the finite set $\Omega_{m_{0}}$. It follows that we can find an infinite set $J \subset \mathbf{N}$ such that $B_{m_{0}, n}$ is independent of $n \in J$. By a diagonal argument we can obtain an infinite set $J \subset \mathbf{N}$ such that if $n>m, n, m \in J, B_{m, n}$ is independent of $n$. We call it $B_{m}$. Now by exchanging the sequence $\left(x_{n}\right)_{n \in \mathbf{N}}$ for $\left(x_{n}\right)_{n \in J}$, we can also assume that the original sequence satisfies these conditions.

Let $\delta=(2-s) /(2-\theta)<1$, and choose $0<\varepsilon<(1-\delta) / 2$. We can assume that $\lim \mathbb{P}_{n}\left(B_{n}\right)$ exists, call it $l$. Thus $l=\lim \mathbb{P}_{n}\left(B_{n}\right) \leq 1$; even more, we can assume $\left|\mathbb{P}_{n}\left(B_{n}\right)-l\right|<\varepsilon$ for every natural number $n$.

Now, for every pair of natural numbers $n>m$

$$
\mathbb{P}_{n}\left(B_{n, m}\right)+\mathbb{P}_{m}\left(B_{m}\right) \leq(2-s) /(2-\theta)=\delta<1 .
$$

Hence $l \leq \delta$ and $l-\varepsilon<\mathbb{P}_{m}\left(B_{m}\right) \leq \delta$.

Therefore we can consider the spaces $\Omega_{n}^{\prime}=\Omega_{n} \backslash B_{n}$ endowed with the measures $\mathbb{P}_{n}^{\prime}=\left(1-\mathbb{P}_{n}\left(B_{n}\right)\right)^{-1} \mathbb{P}_{n}$. This measure is well defined because $\mathbb{P}_{n}\left(B_{n}\right) \leq$ $\delta<1$. Now, for every $m<n$,

$$
\begin{aligned}
\mathbb{P}_{n}^{\prime}\left(B_{n, m} \backslash B_{n}\right) & =\frac{\mathbb{P}_{n}\left(B_{n, m} \backslash B_{n}\right)}{1-\mathbb{P}_{n}\left(B_{n}\right)} \leq \frac{\mathbb{P}_{n}\left(B_{n, m}\right)}{1-\mathbb{P}_{n}\left(B_{n}\right)} \leq \frac{\delta-\mathbb{P}_{m}\left(B_{m}\right)}{1-\mathbb{P}_{n}\left(B_{n}\right)} \\
& \leq \frac{1-\mathbb{P}_{n}\left(B_{n}\right)+\left(\mathbb{P}_{n}\left(B_{n}\right)-\mathbb{P}_{m}\left(B_{m}\right)\right)-(1-\delta)}{1-\mathbb{P}_{n}\left(B_{n}\right)} \\
& \leq 1-\frac{1-\delta-2 \varepsilon}{1-\mathbb{P}_{n}\left(B_{n}\right)} \leq 1-\frac{1-\delta-2 \varepsilon}{1-l+\varepsilon} \\
& =1-\alpha<1 .
\end{aligned}
$$


Hence the conditions in the hypothesis of the D. H. Fremlin and M. Talagrand theorem are satisfied. So, we obtain an infinite set $J \subset \mathbf{N}$ such that, for every $n \in J$,

$$
\mathbb{P}_{n}^{\prime}\left(\bigcup_{\substack{m<n \\ m \in J}}\left(B_{n, m} \backslash B_{n}\right)\right)<1 .
$$

Now, if we put $B_{n}=B_{n, n}$, it follows that

$$
\mathbb{P}_{n}\left(\bigcup_{\substack{m \leq n \\ m \in J}} B_{n, m}\right)<1 .
$$

Thus there exists, for every $n \in J, e_{n} \in \Omega_{n}$ such that

$$
e_{n} \notin \bigcup_{\substack{m \leq n \\ m \in J}} B_{n, m} \text {. }
$$

Now if $n>m$ and $n, m \in J$, we have $e_{n} \notin B_{n, m}$ and $e_{m} \notin B_{m, m}$. Therefore $e_{m} \notin B_{m, n}$. Condition (b) insures that $\left\|e_{n}-e_{m}\right\| \geq \theta$. We have thus found a sequence of points $\left(e_{n}\right)_{n \in J}$ in $A$, such that for every $n \neq m, n, m \in J$, $\left\|e_{n}-e_{m}\right\| \geq \theta>1$ which contradicts $\delta(A)<1$.

\section{ACKNOWLEDGMENT}

I would like to thank Professor L. Rodriguez Piazza for advising me to simplify my first proof and for his important contribution to manage it. I would also like to thank Professor T. Dominguez Benavides for proposing to me the question solved in this paper and for fruitful and patient discussions during its preparation.

\section{REFERENCES}

1. J. Banaś and K. Goebel, Measures of noncompactness in Banach spaces, Marcel Dekker, New York, 1980.

2. G. Darbo, Punti uniti in transformazioni a condominio non compatto, Rend. Sem. Mat. Univ. Padova 24 (1955), 84-92.

3. T. Dominguez Benavides, Some properties of the set and ball measures of non-compactness and applications, J. London Math. Soc. (2) 34 (1986), 120-128.

4. __ Set-contractions and ball-contractions in some classes of spaces, J. Math. Anal. Appl. 136 (1988), 131-140.

5. T. Domínguez Benavides and G. López Acedo, Fixed points of asymptotically contractive mappings J. Math. Anal. Appl. (to appear).

6. J. Elton and E. Odell, The unit ball of every infinite dimensional normed linear space contains $a(1+\varepsilon)$-separated sequence, Colloq. Math. 44 (1981), 105-109.

7. D. H. Fremlin and M. Talagrand, Subgraphs of random graphs, Trans. Amer. Math. Soc. 291 (1985), 551-582.

8. T. Jech, Set theory, Academic Press, New York, 1978. 
9. C. Kottman, Packing and reflexivity in Banach spaces, Trans. Amer. Math. Soc. 150 (1970), 565-576.

10. K. Kuratowski, Sur les espaces complets, Fund. Math. 15 (1930), 301-309.

11. P. Massat, Some properties of condensing maps, Ann. Mat. Pura Appl. (4) 125 (1980), 101-115.

12. B. N. Sadovskii, On a fixed point principle, Funktsional Anal. i Prilozhen 4 (1967), 74-76.

13. J. H. Wells and L. R. Williams, Embeddings and extensions in analysis, Springer-Verlag, Berlin, 1975.

Universidad de Sevilla, Facultad de Matemáticas, Apdo. 1160, 41080-Sevilla, Spain 